\title{
Prognostic value of fibrinogen-to-albumin ratio in patients with gastric cancer receiving first-line chemotherapy
}

\author{
LIQUN ZHANG $^{1,2}$, ZHUO WANG $^{1}$, JIAWEN XIAO $^{2}$, ZHIYAN ZHANG $^{2}$, \\ HAIJING LI $^{2}$, YUANHE WANG ${ }^{1}$, QIAN DONG ${ }^{1}$, HAIYAN PIAO ${ }^{1}$, \\ QIWEI WANG ${ }^{1}$, FEIFEI BI ${ }^{1}$, FANG $\mathrm{LI}^{3}$ and JINGDONG ZHANG ${ }^{1}$
}

\begin{abstract}
${ }^{1}$ Medical Oncology Department of Gastrointestinal Cancer, Liaoning Cancer Hospital and Institute, Cancer Hospital of China Medical University, Shenyang, Liaoning 110042; ${ }^{2}$ Department of Medical Oncology, Shenyang Fifth People's Hospital, Shenyang, Liaoning 110020; ${ }^{3}$ Department of Hepatobiliary Surgery, Liaoning Cancer Hospital and Institute, Cancer Hospital of China Medical University, Shenyang, Liaoning 110042, P.R. China
\end{abstract}

Received March 17, 2020; Accepted June 19, 2020

DOI: $10.3892 / \mathrm{ol} .2020 .11871$

\begin{abstract}
The fibrinogen-to-albumin ratio (FAR), reflecting the systemic coagulation, nutritional and inflammation status of patients, has matured into a prognostic marker for several tumor types. However, only a few studies have assessed the utility of the FAR as a prognostic indicator in patients with advanced gastric cancer (GC) receiving first-line chemotherapy. In the present study, 273 patients with advanced GC who received first-line chemotherapy between January 2014 and January 2019 at the Cancer Hospital of China Medical University (Shenyang, China) were retrospectively analyzed. Using the cut-off values determined by receiver operating characteristic (ROC) analysis, the patients were divided into low-FAR $(\leq 10.03)$ and high-FAR $(>10.03)$, low-fibrinogen $(<3.8 \mathrm{~g} / \mathrm{l})$ and high-fibrinogen $(\geq 3.8 \mathrm{~g} / \mathrm{l})$, and low-albumin $(<40.55 \mathrm{~g} / \mathrm{l})$ and high-albumin $(\geq 40.55 \mathrm{~g} / \mathrm{l})$ groups. The associations of the pretreatment FAR and clinicopathological characteristics with progression-free survival (PFS) and overall survival (OS) were evaluated. In order to estimate the prognostic value of the FAR for patients with poor prognosis or normal fibrinogen and albumin levels, subgroup analyses were performed. The FAR had a higher area under the ROC curve $(0.690 ; 95 \%$ CI: 0.628-0.752; $\mathrm{P}<0.001)$ compared with either fibrinogen or albumin alone, which are common indicators of coagulation, nutritional and inflammatory indices. A high FAR was significantly associated with a more advanced stage, peritoneal metastasis, increased CA72-4 levels and anemia (all $\mathrm{P}<0.05$ ). On survival analysis, a low FAR was
\end{abstract}

Correspondence to: Dr Jingdong Zhang, Medical Oncology Department of Gastrointestinal Cancer, Liaoning Cancer Hospital and Institute, Cancer Hospital of China Medical University, 44 Xiaoheyan Road, Dadong, Shenyang, Liaoning 110042, P.R. China

E-mail: jdzhang@cancerhosp-ln-cmu.com

Key words: fibrinogen-to-albumin ratio, gastric cancer, first-line chemotherapy, prognosis, progression-free survival, overall survival associated with a longer PFS and OS compared with a high FAR (202 vs. 130 days and 376 vs. 270 days, respectively; both $\mathrm{P}<0.001)$, while the hazard ratio (HR) and P-values of the FAR were lower compared with those of fibrinogen and albumin alone on multivariate analysis (PFS: $\mathrm{HR}=0.638,95 \% \mathrm{CI}$ : 0.436-0.932, $\mathrm{P}=0.020$; OS: $\mathrm{HR}=0.568,95 \% \mathrm{CI}: 0.394-0.819$, $\mathrm{P}=0.002)$. Subgroup analysis indicated that among patients with poor prognosis, including multiple metastases, TNM stage IV and abnormal CA72-4 levels, the FAR may be used as an accurate prognostic marker (all $\mathrm{P}<0.05$ ), and may also reliably identify patients with poor prognosis among those with normal fibrinogen and albumin levels (all $\mathrm{P}<0.001$ ). The FAR was indicated to be a valuable marker for predicting PFS and OS in patients with advanced GC receiving first-line chemotherapy and is superior to either fibrinogen or albumin alone.

\section{Introduction}

Gastric cancer (GC) is the third leading cause of cancerassociated mortality worldwide and the high incidence of GC poses a major threat to public health globally (1). At present, GC may be cured by radical resection; however, for patients with advanced-stage disease that can no longer receive radical surgical treatment, the median survival time after treatment with trastuzumab plus chemotherapy is only 13.8 months, the improvement of which poses a challenge to scientists and oncologists (2). Therefore, it is necessary to identify patients with poor prognosis as soon as possible, which will help doctors optimize treatment programs and improve the prognosis and quality of life of patients with advanced GC. The Union for International Cancer Control TNM classification system is one of the most common methods used by clinicians to evaluate the prognosis of patients with GC (3). However, this system has certain limitations, such as the fact that patients with the same tumor stage may have different clinical outcomes $(4,5)$. Therefore, it is imperative to identify other independent prognostic markers for GC to further classify patients who have been staged according to the TNM system into subgroups with good and poor prognosis. The addition of other well-proven markers should enable doctors to treat 
patients with poor prognosis in a more timely and effective manner.

Tumorigenesis is amultifactorial process.Hypercoagulability, inflammation and malnutrition promote the occurrence, development, recurrence and metastasis of tumors and are associated with poor treatment outcomes (6-11). Analysis of existing data has demonstrated that conditions of hypercoagulability, inflammation and poor nutritional status are independent prognostic factors for poor prognosis of patients with GC. As a marker reflecting coagulation function nutritional and the inflammatory status of patients, several studies have used the fibrinogen-to-albumin ratio (FAR) or albumin-to-fibrinogen ratio (AFR) to evaluate the prognosis of various cancer patients, including those with GC, and have found that increased FAR or decreased AFR levels were associated with poor prognosis in cancer patients (12-17). Another critical role of FAR or AFR is to assess the effect of the chemotherapy regimen in a particular population to guide the selection of an optimal therapy (18-20). However, there are currently few reports on the pretreatment FAR being used as a marker to predict progression-free survival (PFS) and overall survival (OS) in patients with GC undergoing first-line chemotherapy.

The aim of the present study was to investigate whether the pretreatment FAR may serve as a multifunctional marker for predicting PFS and OS in patients with advanced GC receiving first-line chemotherapy.

\section{Materials and methods}

Patient characteristics. The data of patients with non-resectable GC from the Cancer Hospital of China Medical University (Shenyang, China) between January 2014 and January 2019 were retrospectively evaluated. All patients included were required to fulfill the following criteria: i) The diagnosis was pathologically confirmed; ii) the TNM stage for patients with GC unable to undergo radical resection was considered only as stage III-IV; iii) the patients included in the data collection received no adjuvant chemoradiotherapy; iv) no severe coagulation disorders, nutritional therapy, acute infections or other inflammatory conditions prior to specimen collection; and v) blood sample collection performed within 1 week prior to the initial first-line chemotherapy. A total of 273 patients with advanced GC meeting the inclusion criteria were included in the present retrospective study, 183 of whom were male and 90 were female. The study protocol was approved by the Cancer Hospital of China Medical University Ethics Committee (Shenyang, China).

Clinical data collection and follow-up. In all patients included in the study, computed tomography, magnetic resonance imaging or other accepted imaging examinations were used to determine the presence of metastatic lymph nodes and organs. The reference range for plasma fibrinogen, albumin and hemoglobin was 2-4, 35-55 and 115-155 g/l, respectively. The accepted normal range for carcinoembryonic antigen, CA19-9 and CA72-4 was 0-5 ng/ml, 0-37 U/ml and 0-6 U/ml, respectively. The FAR was calculated as follows: FAR=fibrinogen $(\mathrm{g} / \mathrm{l}) /$ albumin $(\mathrm{g} / \mathrm{l}) \times 100 \%$.

The primary chemotherapeutic regimens for patients receiving first-line chemotherapy are as follows: SOX (oxali-

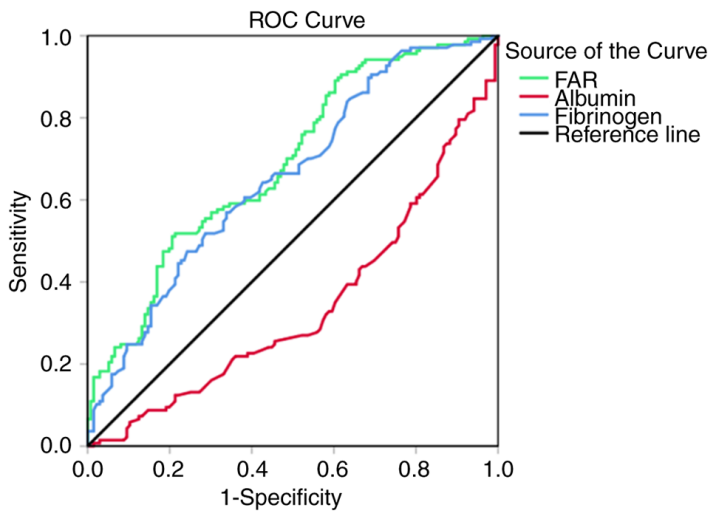

Figure 1. ROC curve analyses for prognostic indicators in patients with advanced gastric cancer. ROC, receiver operating characteristic; FAR, fibrinogen-to-albumin ratio.

platin + S1)/CapeOX (oxaliplatin + capecitabine), FOLFOX (oxaliplatin + leucovorin +5 -fluorouracil) and DCF (docetaxel + cisplatin +5 -fluorouracil)/DOF (docetaxel + oxaliplatin + 5-fluorouracil). A total of 137 patients received SOX/CapeOX regimen, 49 patients received FOLFOX regimen, 36 patients received $\mathrm{DCF} / \mathrm{DOF}$ regimen and 51 patients received $\mathrm{S} 1$ or capecitabine monotherapy. The efficacy of treatment was evaluated every 2-3 cycles. The Response Evaluation Criteria in Solid Tumors version 1.1 were employed to estimate the response to chemotherapy (21). Patients with first-line chemotherapy failure were followed up every 2-3 months. The latest follow-up date was December 2019. PFS and OS were considered as the primary endpoints. PFS was defined as the time from start of first-line chemotherapy until the first observed progression or the last follow-up visit without progression, while OS was defined as the time from the beginning of first-line chemotherapy to death from any cause or the end of follow-up.

Statistical analysis. Data analysis was performed using SPSS v21 (IBM, Corp.). Receiver operating characteristic (ROC) curve analysis was applied to calculate the cut-off values of the FAR, fibrinogen and albumin. Fisher's exact test and the $\chi^{2}$ test were employed to evaluate the association between the FAR and the clinicopathological characteristics. Univariate and multivariate data analyses were conducted to identify potential predictors of PFS and OS. The Kaplan-Meier method was used to construct survival curves. The survival of patients was compared with the log-rank test. $\mathrm{P}<0.05$ was considered to indicate a statistical significant difference.

\section{Results}

Optimal cut-off value of the FAR. In addition to the FAR, the present study also assessed fibrinogen and albumin to determine whether the FAR is superior to either fibrinogen or albumin alone in predicting the prognosis of patients with GC. The area under the ROC curve (AUC) is considered to indicate the overall diagnostic power of a model, with a larger AUC indicating better diagnostic power of the prognostic predictor. In order to assess the ability of the FAR to discriminate between patients with advanced GC with different prognosis, 
Table I. Association between pretreatment FAR and clinicopathological characteristics.

\begin{tabular}{|c|c|c|c|c|}
\hline Variable & All & Low FAR & High FAR & P-value \\
\hline Total & $273(100.0)$ & $175(64.10)$ & $98(35.90)$ & \\
\hline Age (years) & & & & 0.057 \\
\hline$<60$ & $135(49.45)$ & 79 (45.14) & $56(57.14)$ & \\
\hline$\geq 60$ & $138(50.55)$ & $96(54.86)$ & $42(42.86)$ & \\
\hline Sex & & & & 0.726 \\
\hline Male & $183(67.03)$ & $116(66.29)$ & $67(68.37)$ & \\
\hline Female & $90(32.97)$ & $59(33.71)$ & $31(31.63)$ & \\
\hline Body mass index $\left(\mathrm{kg} / \mathrm{m}^{2}\right)$ & & & & 0.965 \\
\hline$<18.5$ or $>25$ & $72(26.37)$ & $46(26.29)$ & $26(26.53)$ & \\
\hline $18.5-25$ & $201(73.63)$ & $129(73.71)$ & $72(73.47)$ & \\
\hline ECOG PS score & & & & 0.695 \\
\hline $0-1$ & $228(83.52)$ & $145(82.86)$ & $83(84.69)$ & \\
\hline$>1$ & $45(16.48)$ & $30(17.14)$ & $15(15.31)$ & \\
\hline Histological differentiation & & & & 0.050 \\
\hline High/moderate & $72(26.37)$ & $53(30.29)$ & $19(19.39)$ & \\
\hline Poor/mucinous & $201(73.63)$ & $122(69.71)$ & $79(80.61)$ & \\
\hline Number of organs with metastasis & & & & 0.486 \\
\hline $0-1$ & $180(65.93)$ & $118(67.43)$ & $62(63.27)$ & \\
\hline$>1$ & $93(34.07)$ & $57(32.57)$ & $36(36.73)$ & \\
\hline Peritoneal metastasis & & & & 0.010 \\
\hline Yes & $80(29.30)$ & $42(24.00)$ & $38(38.78)$ & \\
\hline No & $193(70.70)$ & $133(76.00)$ & $60(61.22)$ & \\
\hline TNM stage & & & & 0.025 \\
\hline III & $53(19.41)$ & $41(23.43)$ & $12(12.24)$ & \\
\hline IV & $220(80.59)$ & $134(76.57)$ & $86(87.76)$ & \\
\hline $\mathrm{CEA}(\mathrm{ng} / \mathrm{ml})$ & & & & 0.691 \\
\hline$\leq 5$ & $152(55.68)$ & $99(56.57)$ & $53(54.08)$ & \\
\hline$>5$ & $121(44.32)$ & $76(43.43)$ & $45(45.92)$ & \\
\hline CA19-9 (U/ml) & & & & 0.264 \\
\hline$\leq 37$ & $168(61.54)$ & $112(64.00)$ & $56(57.14)$ & \\
\hline$>37$ & $105(38.46)$ & $63(36.00)$ & $42(42.86)$ & \\
\hline CA72-4 (U/ml) & & & & 0.027 \\
\hline$\leq 6$ & 116 (42.49) & $83(47.43)$ & $33(33.67)$ & \\
\hline$>6$ & $157(57.51)$ & $92(52.57)$ & $65(66.33)$ & \\
\hline Hemoglobin (g/l) & & & & 0.031 \\
\hline$<115$ & $105(38.46)$ & $59(33.71)$ & $46(46.94)$ & \\
\hline$\geq 115$ & $168(61.54)$ & $116(66.29)$ & $52(53.06)$ & \\
\hline
\end{tabular}

Values are expressed as n (\%). FAR, fibrinogen-to-albumin ratio; ECOG PS, Eastern Cooperative Oncology Group performance status.

ROC curve analysis was performed to determine the optimal cut-off values of the FAR, fibrinogen and albumin, and the AUC values of these three indicators were further compared. The median overall survival of 339 days was used as the fixed variable and the levels of FAR, fibrinogen and albumin as the test variables to determine the cut-off values. The optimal cut-off values of the FAR, fibrinogen and albumin were 10.033, 3.795 and 40.550, respectively. The FAR had a higher AUC value $(0.690 ; 95 \%$ CI: $0.628-0.752 ; \mathrm{P}<0.001)$ compared with fibrinogen $(0.657$; 95\% CI: $0.593-0.721 ; \mathrm{P}<0.001)$ and albumin (0.660; 95\% CI: 0.596-0.724; P<0.001; Fig. 1), which indicated that the FAR is likely superior to fibrinogen and albumin alone in predicting the prognosis of patients with GC. The cut-off values of the FAR, fibrinogen and albumin were set as $10.03,3.80$ and 40.55 , respectively, in the further analyses and patients with FAR $>10.03$, fibrinogen $\geq 3.80$ and albumin $\geq 40.55$ comprised the high-FAR group, whereas the remaining patients comprised the low-FAR group. 
Table II. Associations of PFS with FAR and other clinicopathological factors.

\begin{tabular}{|c|c|c|c|c|c|c|}
\hline \multirow[b]{2}{*}{ Factor } & \multicolumn{3}{|c|}{ Univariate analysis } & \multicolumn{3}{|c|}{ Multivariate analysis } \\
\hline & HR & $95 \% \mathrm{CI}$ & P-value & HR & $95 \% \mathrm{CI}$ & P-value \\
\hline Age $>60$ years & 0.988 & $0.778-1.255$ & 0.921 & & & \\
\hline Male sex & 1.140 & $0.884-1.471$ & 0.313 & & & \\
\hline \multicolumn{7}{|l|}{ Body mass index } \\
\hline$<18.5$ or $>25 \mathrm{~kg} / \mathrm{m}^{2}$ & 0.940 & $0.717-1.231$ & 0.651 & & & \\
\hline ECOG PS score $>1$ & 1.295 & $0.940-1.784$ & 0.114 & 1.164 & $0.832-1.629$ & 0.376 \\
\hline Histological differentiation, poor/mucinous & 1.144 & $0.873-1.500$ & 0.329 & & & \\
\hline Metastasis in $>1$ organ & 1.464 & $1.138-1.883$ & 0.003 & 1.232 & $0.920-1.651$ & 0.162 \\
\hline Peritoneal metastasis & 1.813 & $1.388-2.367$ & $<0.001$ & 1.370 & $1.011-1.857$ & 0.042 \\
\hline TNM stage IV & 1.412 & $1.042-1.913$ & 0.026 & 1.060 & $0.759-1.481$ & 0.731 \\
\hline $\mathrm{CEA}>5 \mathrm{ng} / \mathrm{ml}$ & 1.162 & $0.913-1.480$ & 0.223 & & & \\
\hline CA19-9 $>37 \mathrm{U} / \mathrm{ml}$ & 0.972 & $0.761-1.243$ & 0.823 & & & \\
\hline CA72-4 >6 U/ml & 1.506 & $1.178-1.926$ & 0.001 & 1.375 & $1.068-1.770$ & 0.013 \\
\hline Hemoglobin <115 g/l & 0.919 & $0.718-1.176$ & 0.502 & & & \\
\hline Fibrinogen $<3.8 \mathrm{~g} / 1$ & 0.567 & $0.441-0.729$ & $<0.001$ & 0.805 & $0.564-1.149$ & 0.231 \\
\hline Albumin $\geq 40.55 \mathrm{~g} / \mathrm{l}$ & 0.697 & $0.547-0.887$ & 0.003 & 0.770 & 0.595-0.998 & 0.048 \\
\hline FAR $\leq 10.03$ & 0.468 & $0.360-0.608$ & $<0.001$ & 0.638 & $0.436-0.932$ & 0.020 \\
\hline
\end{tabular}

FAR, fibrinogen-to-albumin ratio; PFS, progression-free survival; ECOG PS, Eastern Cooperative Oncology Group performance status; HR, hazard ratio.

Association of clinicopathological characteristics with the FAR. Of the 273 patients included in the present study, 183 $(67.03 \%)$ were male and $90(32.97 \%)$ were female. The median age was 60 years (range, 52-65 years) and the median body mass index was $21.6 \mathrm{~kg} / \mathrm{m}^{2}$ (range, $19.6-23.6 \mathrm{~kg} / \mathrm{m}^{2}$ ). There were $80(29.30 \%)$ patients with peritoneal metastasis and $220(80.59 \%)$ patients with stage IV disease. Patients with a CA72-4 level $>6 \mathrm{U} / \mathrm{ml}$ and hemoglobin $<115 \mathrm{~g} / \mathrm{l}$ accounted for 57.51 and $38.46 \%$ of the total, respectively (Table I).

There were certain differences in the clinicopathological characteristics between the low-FAR and the high-FAR groups. An elevated FAR was significantly associated with peritoneal carcinomatosis $(\mathrm{P}=0.010)$, stage IV cancer $(\mathrm{P}=0.025)$, increased CA72-4 levels $(\mathrm{P}=0.027)$ and anemia $(\mathrm{P}=0.031)$. The clinicopathological characteristics of the patients are further described in detail in Table I.

Prognostic factors indicating patient survival. As presented in Table II, univariate analyses revealed that metastasis in $>1$ organ $(\mathrm{P}=0.003)$, peritoneal metastasis $(\mathrm{P}<0.001)$, TNM stage IV $(\mathrm{P}=0.026)$ and increased $\mathrm{CA} 72-4$ levels $(\mathrm{P}=0.001)$ were significantly associated with shorter PFS, while increased albumin $(\mathrm{P}=0.003)$ and reduced fibrinogen $(\mathrm{P}<0.001)$ and FAR $(\mathrm{P}<0.001)$ levels were significantly associated with longer PFS. On multivariate analysis, only peritoneal metastasis $(\mathrm{P}=0.042), \mathrm{CA} 72-4$ levels $(\mathrm{P}=0.013)$, albumin $(\mathrm{P}=0.048)$ and $\mathrm{FAR}(\mathrm{P}=0.020)$ were indicated to be independently associated with PFS. As the $\mathrm{P}$-value of FAR $(\mathrm{P}=0.020)$ was lower compared with that of fibrinogen $(\mathrm{P}=0.231)$ and albumin $(\mathrm{P}=0.048)$ alone, the FAR was considered as significantly more effective than either fibrinogen or albumin alone in predicting PFS in patients with GC.
As presented in Table III, metastasis to $>1$ organ $(\mathrm{P}=0.030)$, peritoneal metastasis $(\mathrm{P}<0.001)$ and CA72-4 levels $>6 \mathrm{U} / \mathrm{ml}$ $(\mathrm{P}=0.033)$ were identified as risk factors adversely affecting the OS of patients with GC, while an age of $>60$ years $(\mathrm{P}=0.040)$, fibrinogen $<3.8 \mathrm{~g} / \mathrm{l}(\mathrm{P}<0.001)$, albumin $\geq 40.55 \mathrm{~g} / 1$ $(\mathrm{P}<0.001)$ and FAR $\leq 10.03(\mathrm{P}<0.001)$ were indicated to be protective factors favorably affecting the OS of patients with GC on univariate analysis. The results of the multivariate analysis demonstrated that the OS of patients with GG was independently associated with peritoneal metastasis $(\mathrm{P}=0.019)$, albumin $(\mathrm{P}=0.016)$ and FAR $(\mathrm{P}=0.002)$. FAR $(\mathrm{P}=0.002)$ was superior to fibrinogen $(\mathrm{P}=0.938)$ and albumin $(\mathrm{P}=0.016)$ in predicting the OS of patients with GC.

Survival analysis according to pretreatment FAR. The median PFS and OS of the patients included in the present study were 174 and 339 days, respectively. The low-FAR group had longer median PFS and OS compared with the high-FAR group (202 vs. 130 days, $\mathrm{P}<0.001$; and 376 vs. 270 days, $\mathrm{P}<0.001$, respectively; Fig. 2).

Survival analysis for the FAR according to the prognostic factor subtypes of $G C$. Previous studies demonstrated that distant metastasis, peritoneal infiltration, TNM stage IV disease and increased CA72-4 levels are crucial characteristics indicating unfavorable prognosis in patients with GC (22-24). Therefore, a subgroup analysis was performed to investigate whether the pretreatment FAR is able to further predict the outcomes of patients with GC with poor prognosis (Fig. 3). The results suggested improved PFS and OS in the low-FAR group within the subgroups with $>1$ metastatic organ $(\mathrm{P}=0.001$ and 
Table III. Associations of OS with FAR and other clinicopathological factors.

\begin{tabular}{|c|c|c|c|c|c|c|}
\hline \multirow[b]{2}{*}{ Factor } & \multicolumn{3}{|c|}{ Univariate analysis } & \multicolumn{3}{|c|}{ Multivariate analysis } \\
\hline & HR & $95 \%$ CI & P-value & HR & $95 \% \mathrm{CI}$ & P-value \\
\hline Age $>60$ years & 0.778 & $0.612-0.989$ & 0.040 & 0.869 & $0.674-1.120$ & 0.278 \\
\hline Male sex & 1.136 & $0.881-1.465$ & 0.324 & & & \\
\hline \multicolumn{7}{|l|}{ Body mass index } \\
\hline$<18.5$ or $>25 \mathrm{~kg} / \mathrm{m}^{2}$ & 1.014 & $0.774-1.329$ & 0.921 & & & \\
\hline ECOG PS score >1 & 1.304 & $0.946-1.798$ & 0.105 & 1.196 & $0.858-1.668$ & 0.291 \\
\hline Histological differentiation, poor/mucinous & 1.268 & $0.968-1.661$ & 0.084 & 1.096 & $0.832-1.444$ & 0.516 \\
\hline Metastasis in $>1$ organ & 1.326 & $1.028-1.709$ & 0.030 & 1.166 & 0.884-1.537 & 0.277 \\
\hline Peritoneal metastasis & 1.932 & $1.478-2.524$ & $<0.001$ & 1.441 & $1.063-1.955$ & 0.019 \\
\hline TNM stage IV & 1.188 & $0.879-1.605$ & 0.263 & & & \\
\hline $\mathrm{CEA}>5 \mathrm{ng} / \mathrm{ml}$ & 1.123 & $0.884-1.428$ & 0.342 & & & \\
\hline CA19-9 >37 U/ml & 1.072 & $0.839-1.369$ & 0.578 & & & \\
\hline CA72-4 >6 U/ml & 1.301 & $1.021-1.656$ & 0.033 & 1.224 & $0.951-1.574$ & 0.116 \\
\hline Hemoglobin <115 g/l & 0.932 & $0.729-1.192$ & 0.575 & & & \\
\hline Fibrinogen $<3.8 \mathrm{~g} / 1$ & 0.628 & $0.490-0.804$ & $<0.001$ & 0.986 & 0.695-1.399 & 0.938 \\
\hline Albumin $\geq 40.55 \mathrm{~g} / 1$ & 0.635 & $0.498-0.810$ & $<0.001$ & 0.725 & $0.559-0.941$ & 0.016 \\
\hline FAR $\leq 10.03$ & 0.463 & $0.358-0.599$ & $<0.001$ & 0.568 & 0.394-0.819 & 0.002 \\
\hline
\end{tabular}

FAR, fibrinogen-to-albumin ratio; OS, overall survival; ECOG PS, Eastern Cooperative Oncology Group performance status; HR, hazard ratio.
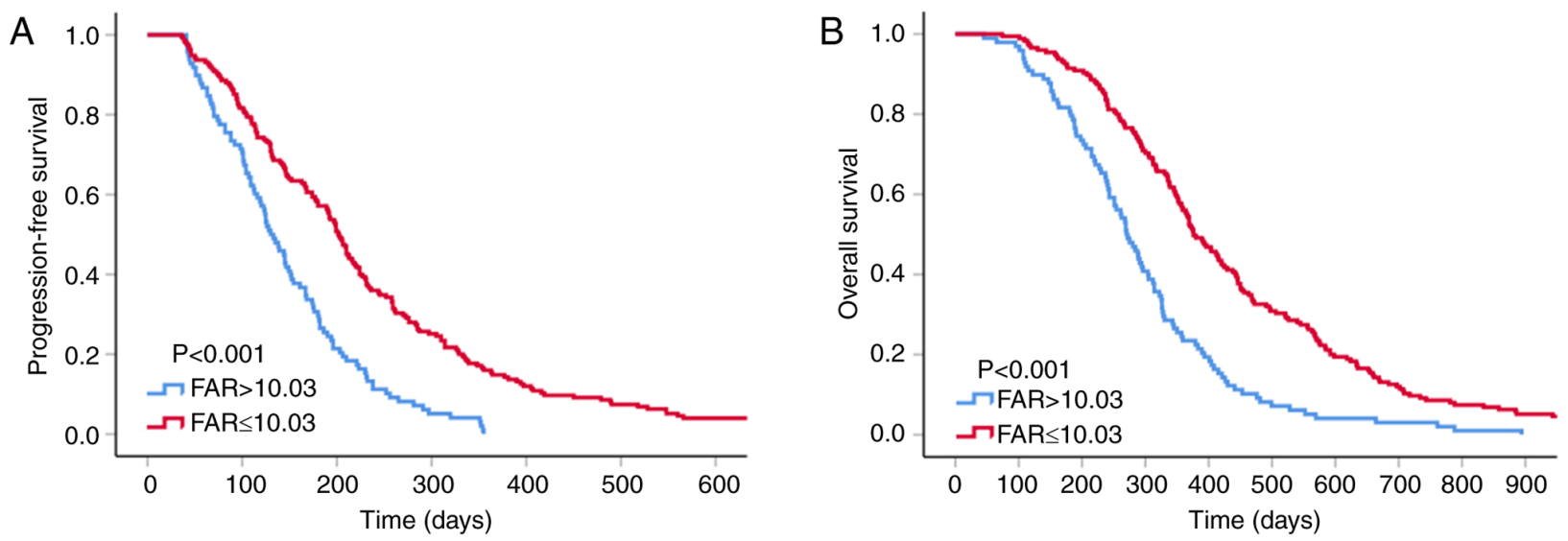

Figure 2. Kaplan-Meier curves for (A) progression-free and (B) overall survival according to the cut-off value of FAR in patients. FAR, fibrinogen-to-albumin ratio.

$\mathrm{P}<0.001)$, stage IV cancer (all $\mathrm{P}<0.001)$ and elevated CA72-4 levels $(\mathrm{P}=0.001$ and $\mathrm{P}<0.001)$. Although $\mathrm{GC}$ patients with low FAR within the peritoneal invasion subgroup tended to have longer PFS and OS, there was no statistical insignificance (all $\mathrm{P}>0.05)$ and this result may have been obtained due to the small sample size $(\mathrm{n}=80)$.

For patients with normal albumin and fibrinogen levels, clinicians may be prone to overlooking the coagulation, inflammation and nutritional status that may indicate the prognosis of such patients. Thus, a subgroup analysis was performed to investigate whether the FAR is able to provide a clue as to the prognosis of patients with normal albumin and fibrinogen indices. The results suggested that low FAR within normal plasma fibrinogen and albumin level subgroups of patients with GC was an indicator of longer PFS and OS (all $\mathrm{P}<0.001$; Fig. 4).

\section{Discussion}

Previous studies have reported the importance of the FAR in predicting the prognosis of different types of cancer (25-29). Zhang et al (30) indicated that a reduced AFR was an independent predictor of poor OS in surgical stage II and III GC. In the present study, it was determined that patients with GC and a high FAR had shortened OS. In other words, the previous and the present study suggested that patients with elevated fibrinogen levels and decreased albumin levels had poor prognosis. Therefore, the conclusion regarding the 

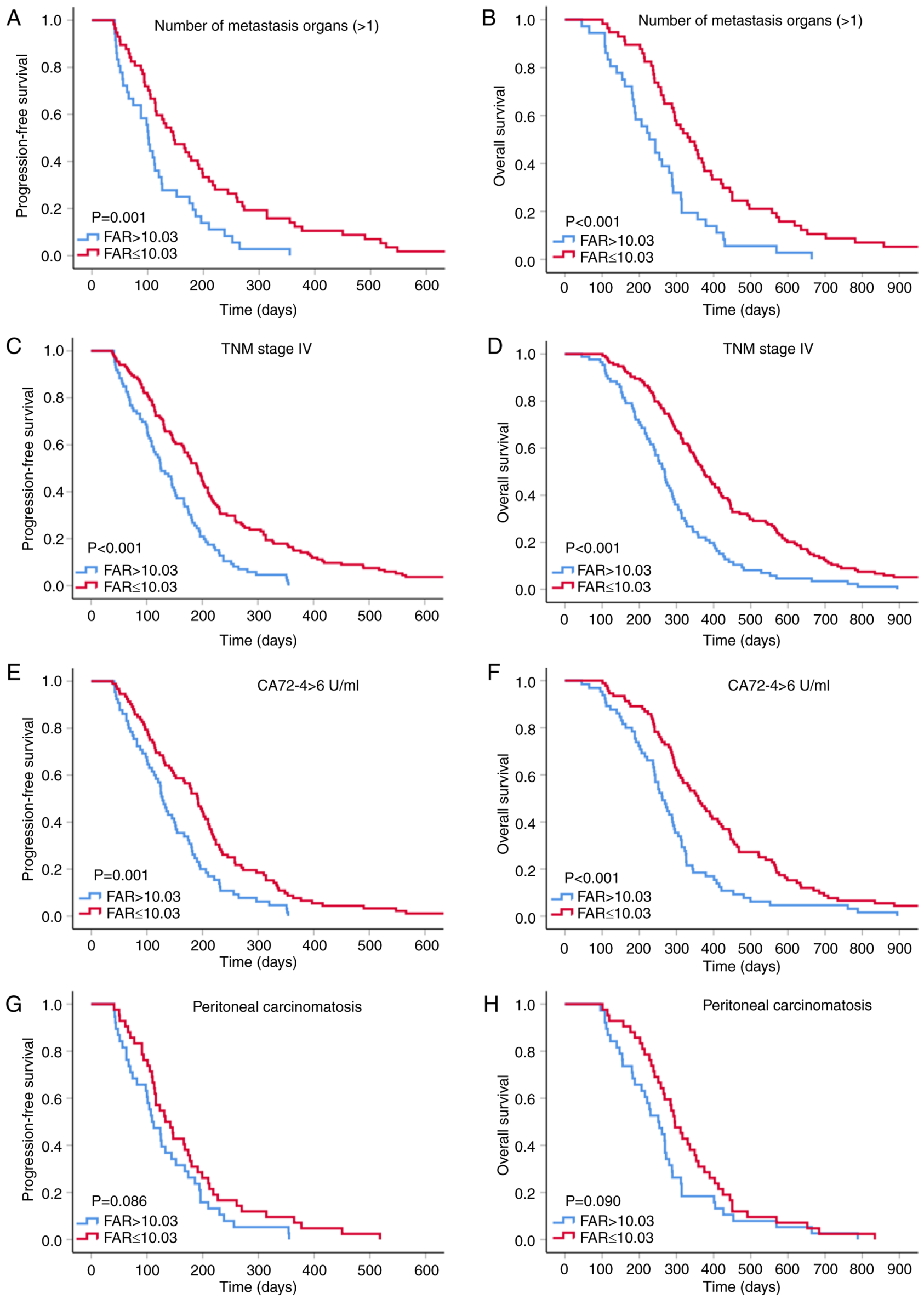

Figure 3. Kaplan-Meier curves for FAR according to various risk groups. (A) PFS and (B) OS for number of metastasis organs (>1); (C) PFS and (D) OS for patients with TNM stage IV; (E) PFS and (F) OS for patients with CA72-4 >6 U/ml; (G) PFS and (H) OS for patients with peritoneal carcinomatosis. FAR, fibrinogen-to-albumin ratio; PFS, progression-free survival; OS, overall survival. 

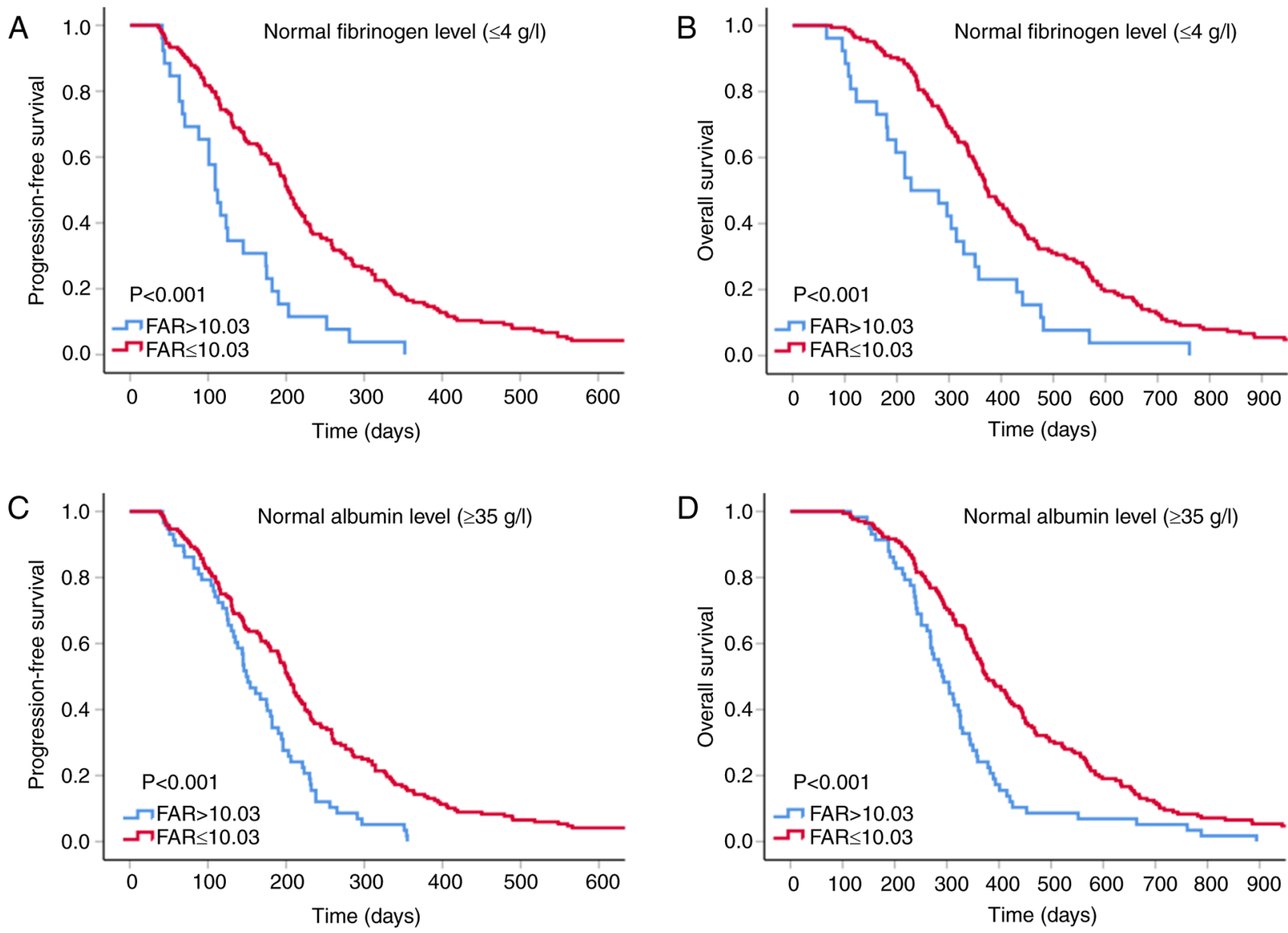

Figure 4. Kaplan-Meier curves for FAR for patients with normal levels of fibrinogen and albumin. (A) PFS and (B) OS for patients with normal fibrinogen; (C) PFS and (D) OS for patients with normal albumin levels. FAR, fibrinogen-to-albumin ratio; PFS, progression-free survival; OS, overall survival.

prognostic value of AFR and FAR for predicting the OS of patients with GC in these two studies are similar. However, the patient characteristics of the present cohort are different from those in the previous study: The patients of the present study had stage III-IV unresectable GC and all patients received first-line chemotherapy. To the best of our knowledge, the present study was the first study to indicate that the FAR may be an independent predictor of first-line chemotherapy-associated PFS and long-term OS in patients with advanced GC. Furthermore, the prognostic value of the FAR was more powerful compared with that of either fibrinogen or albumin alone. The most noteworthy result of the present study was that the subgroup analysis results demonstrated that the FAR may be a cost-effective and accessible marker for patients with poor prognosis to further predict the clinical outcomes of these populations. Furthermore, the FAR may be a reliable indicator for predicting the prognosis of patients with normal albumin and fibrinogen levels that may otherwise not draw the physicians' attention. Therefore, physicians may be able to formulate personalized treatment patterns for patients with GC with different prognosis by effectively applying the FAR.

There is increasing and credible evidence that cancerassociated hypercoagulability, inflammation and malnutrition are highly prevalent among cancer patients. These abnormal conditions may weaken the response of tumor patients to treat- ment and promote the occurrence, development, metastasis and exacerbation of cancer (31-36). The potential mechanism may be as follows: First, patients with malignant tumors may occasionally be in a hypercoagulable state, which may manifest as venous thromboembolism and disseminated intravascular coagulation. Although thrombosis may not be common among patients with advanced cancer, systemic hypercoagulability is frequently observed. The coagulation cascade has a crucial role in tumor growth. Components involved in the hypercoagulable state provide a stable framework for the tumor extracellular matrix, which sets the conditions for angiogenesis, adhesion, migration and invasion of tumor cells. The interaction of coagulation cytokines may also impede the cytotoxicity of immune cells against tumor cells (37). Furthermore, cytokines may also induce tumor cell proliferation and invasion by mediating the adhesion between leukocytes and endothelial cells, which promotes inflammation (38).

Second, inflammation is a hallmark of cancer (39). Inflammation may lead to mutagenesis, predisposing to the accumulation of mutations in tumor protein 53 and other cancer-associated genes and chronic inflammation that induces tissue damage may disrupt the barrier function, expose the stem cell region to environmental carcinogens or facilitate stem cells to be eroded by genotoxic compounds, which may trigger tumor formation (40). Kim et al (41) reported that tumor necrosis factor (TNF)- $\alpha$ and Toll-like receptor 
family member-2 (TLR2) may be essential for cancer metastasis. Cancer cells may trigger macrophage activity, which promotes the production of TNF- $\alpha$ by activating TLR2. The extracellular matrix proteoglycan versican, which is elevated in tumors, augments metastatic cancer growth by triggering TLR 2 complexes and causing TNF- $\alpha$ release. These factors create an inflammatory microenvironment, which promotes metastatic growth (41).

Finally, malnutrition is universal among cancer patients receiving oncological treatment. Patients suffering from malnutrition have lower tumor treatment completion rates, poorer quality of life and more complications, which ultimately leads to lower survival rates (42). Malnutrition may cause fluctuation in the bone marrow stromal microenvironment, debilitate hematopoiesis and trigger thymic atrophy through apoptosis of immature CD4/CD8 double-positive thymocytes, thereby promoting a decline in immunity, which facilitates the proliferation of tumor cells (43).

Previous studies have demonstrated that anti-coagulant therapy, anti-inflammatory treatment and nutritional support may reduce susceptibility to cancer, prevent disease exacerbation and improve the clinical outcome (44-46). As a reliable prognostic indicator, the FAR relies on fibrinogen and albumin. Fibrinogen is an acute-phase protein produced by the liver and its plasma levels increase under hypercoagulable and inflammatory conditions (47-49). A large body of evidence indicated that fibrinogen-related coagulation dysfunction is tightly linked to cancer angiogenesis, invasion, progression and metastasis $(50,51)$. Reliable data have demonstrated that elevated fibrinogen levels are associated with poor prognosis of patients with cancer, including GC $(52,53)$. Albumin is also produced by liver cells. The tumor-associated proinflammatory cytokines TNF- $\alpha$ and interleukin- 6 are able to inhibit albumin production by hepatocytes (54). The decrease in plasma albumin levels indicates a high degree of inflammation, poor nutritional status and poor treatment efficacy $(55,56)$. Albumin has also been investigated for its potential value in predicting shorter survival in a number of cancer types, including GC $(57,58)$.

As mentioned above, albumin and fibrinogen are important prognostic indicators for patients with GC, but their accuracy may be compromised by certain factors, such as dehydration or fluid retention. The results of the present ROC curve analysis demonstrated that the AUC of the FAR (0.690) was higher compared with that of fibrinogen (0.657) and albumin (0.660), and the P-value of FAR (PFS: $\mathrm{P}=0.020$; OS: $\mathrm{P}=0.002$ ) was lower compared with that of fibrinogen (PFS: $\mathrm{P}=0.231$; OS: $\mathrm{P}=0.938$ ) and albumin (PFS: $\mathrm{P}=0.048$; $\mathrm{OS}: \mathrm{P}=0.016$ ) on multivariate analyses, which indicated that the prognostic value of the FAR was superior to that of either fibrinogen or albumin alone.

Based on the results of the present study, clinicians may use the FAR to distinguish patients with poor prognosis and personalize treatment to improve their quality of life and prolong survival. There were certain limitations to the present study. First, as this was a single-center retrospective study, the usefulness of the FAR requires verification by multicenter large-scale studies. Furthermore, a total of 273 patients included in the present study, which is a small sample size and may be insufficient to draw definitive conclusions.

In conclusion, the FAR prior to first-line chemotherapy may help identify specific patient populations that are likely to benefit from chemotherapy and is an independent predictor of PFS and OS; therefore, it may be used as an innovative, dependable prognostic index for patients with advanced-stage GC.

\section{Acknowledgements}

Not applicable.

\section{Funding}

The present study was supported by the National Key R\&D Program of China (grant no. 2018YFC1311600), the Scientific Research Foundation for the Introduction of Talents, Liaoning Cancer Hospital \& Institute (grant no. Z1702), the Science and Technology Planning Project of Liaoning Province of China (grant no. 201800449) and the Science and Technology Planning Project of Shenyang (grant no. 191124088).

\section{Availability of data and materials}

The datasets used and/or analyzed during the current study are available from the corresponding author on reasonable request.

\section{Authors' contributions}

LZ analyzed the patient data and was a major contributor in writing the manuscript. LZ, ZW, JX, ZZ, HL, YW, QD, HP, $\mathrm{QW}, \mathrm{FB}, \mathrm{FL}$ and $\mathrm{JZ}$ performed the retrospective analysis of patients. All authors read and approved the final manuscript.

\section{Ethics approval and consent to participate}

This project was approved by the Ethical Committee of the Cancer Hospital of China Medical University (Shenyang, China). Due to the retrospective nature of this study the Ethics Committee waived written informed consent of the included patients.

\section{Patient consent for publication}

Not applicable.

\section{Competing interests}

All the authors declare that they have no competing interests.

\section{References}

1. Bray F, Ferlay J, Soerjomataram I, Siegel RL, Torre LA and Jemal A: Global cancer statistics 2018: GLOBOCAN estimates of incidence and mortality worldwide for 36 cancers in 185 countries. CA Cancer J Clin 68: 394-424, 2018.

2. Bang YJ, Van Cutsem E, Feyereislova A, Chung HC, Shen L, Sawaki A, Lordick F, Ohtsu A, Omuro Y, Satoh T, et al: Trastuzumab in combination with chemotherapy versus chemotherapy alone for treatment of HER2-positive advanced gastric or gastro-oesophageal junction cancer (ToGA): A phase 3, open-label, randomised controlled trial. Lancet 376: 687-697, 2010.

3. Fang C, Wang W, Deng JY, Sun Z, Seeruttun SR, Wang ZN, $\mathrm{Xu} \mathrm{HM}$, Liang $\mathrm{H}$ and Zhou ZW: Proposal and validation of a modified staging system to improve the prognosis predictive performance of the 8th AJCC/UICC pTNM staging system for gastric adenocarcinoma: A multicenter study with external validation. Cancer Commun (Lond) 38: 67, 2018 
4. Yoon JY, Sigel K, Martin J, Jordan R, Beasley MB, Smith C, Kaufman A, Wisnivesky J and Kim MK: Evaluation of the prognostic significance of TNM staging guidelines in lung carcinoid tumors. J Thorac Oncol 14: 184-192, 2019.

5. Shao Y, Geng Y, Gu W, Ning Z, Huang J, Pei H and Jiang J: Assessment of lymph node ratio to replace the pn categories system of classification of the tnm system in esophageal squamous cell carcinoma. J Thorac Oncol 11: 1774-1784, 2016.

6. Khorana AA and Fine RL: Pancreatic cancer and thromboembolic disease. Lancet Oncol 5: 655-663, 2004.

7. Miller GJ, Bauer KA, Howarth DJ, Cooper JA, Humphries SE and Rosenberg RD: Increased incidence of neoplasia of the digestive tract in men with persistent activation of the coagulant pathway. J Thromb Haemost 2: 2107-2114, 2004.

8. Balkwill $\mathrm{F}$ and Mantovani A: Inflammation and cancer: Back to Virchow? Lancet 357: 539-545, 2001

9. Mantovani A, Allavena P, Sica A and Balkwill F: Cancer-related inflammation. Nature 454: 436-444, 2008.

10. Arends J,Bodoky G, Bozzetti F,Fearon K, Muscaritoli M, Selga G, van Bokhorst-de van der Schueren MA, von Meyenfeldt M; DGEM (German Society for Nutritional Medicine); Zürcher G, et al: ESPEN guidelines on enteral nutrition: Non-surgical oncology. Clin Nutr 25: 245-259, 2006.

11. Bozzetti F, Arends J, Lundholm K, Micklewright A, Zurcher G and Muscaritoli M; ESPEN: ESPEN Guidelines on parenteral nutrition: Non-surgical oncology. Clin Nutr 28: 445-454, 2009.

12. Xu Q, Yan Y, Gu S, Mao K, Zhang J, Huang P, Zhou Z, Chen Z, Zheng S, Liang J, et al: A novel inflammation-based prognostic score: The fibrinogen/albumin ratio predicts prognoses of patients after curative resection for hepatocellular carcinoma. J Immunol Res 2018: 4925498, 2018.

13. Wang YY, Liu ZZ, Xu D, Liu M, Wang K and Xing BC: Fibrinogen-albumin ratio index (fari): A more promising inflammation-based prognostic marker for patients undergoing hepatectomy for colorectal liver metastases. Ann Surg Oncol 26: 3682-3692, 2019

14. Xu WY, Zhang HH, Xiong JP, Yan XB, Bai Y, Lin JZ, Long JY, Zheng YC, Zhao HT and Sang XT: Prognostic significance of the fibrinogen-to-albumin ratio in gallbladder cancer patients. World J Gastroenterol 24: 3281-3292, 2018

15. Li SQ, Jiang YH, Lin J, Zhang J, Sun F, Gao QF, Zhang L, Chen QG, Wang XZ and Ying HQ: Albumin-to-fibrinogen ratio as a promising biomarker to predict clinical outcome of non-smal cell lung cancer individuals. Cancer Med 7: 1221-1231, 2018.

16. Zou YX, Qiao J, Zhu HY, Lu RN, Xia Y, Cao L, Wu W, Jin H, Liu WJ, Liang JH, et al: Albumin-to-fibrinogen ratio as an independent prognostic parameter in untreated chronic lymphocytic leukemia: A retrospective study of 191 cases. Cancer Res Treat 51: 664-671, 2019

17. You X, Zhou Q, Song J, Gan L, Chen J and Shen H: Preoperative albumin-to-fibrinogen ratio predicts severe postoperative complications in elderly gastric cancer subjects after radical laparoscopic gastrectomy. BMC Cancer 19: 931, 2019.

18. Yu W, Ye Z, Fang X, Jiang $X$ and Jiang Y: Preoperative albumin-to-fibrinogen ratio predicts chemotherapy resistance and prognosis in patients with advanced epithelial ovarian cancer. J Ovarian Res 12: 88, 2019.

19. Ying J, Zhou D, Gu T, Huang J and Liu H: Pretreatment albumin/fibrinogen ratio as a promising predictor for the survival of advanced non small-cell lung cancer patients undergoing first-line platinum-based chemotherapy. BMC Cancer 19: 288, 2019.

20. Zhang L, Zhang J, Wang Y, Dong Q, Piao H, Wang Q, Zhou Y and Ding Y: Potential prognostic factors for predicting the chemotherapeutic outcomes and prognosis of patients with metastatic colorectal cancer. J Clin Lab Anal 33: e22958, 2019.

21. Eisenhauer EA, Therasse P, Bogaerts J, Schwartz LH, Sargent D, Ford R, Dancey J, Arbuck S, Gwyther S, Mooney M, et al: New response evaluation criteria in solid tumours: Revised RECIST guideline (version 1.1). Eur J Cancer 45: 228-247, 2009.

22. Singh P, Toom S and Huang Y: Anti-claudin 18.2 antibody as new targeted therapy for advanced gastric cancer. J Hematol Onco 10: 105, 2017.

23. Thomassen I, van Gestel YR, van Ramshorst B, Luyer MD, Bosscha K, Nienhuijs SW, Lemmens VE and de Hingh IH: Peritoneal carcinomatosis of gastric origin: A population-based study on incidence, survival and risk factors. Int J Cancer 134 622-628, 2014

24. Shimada H, Noie T, Ohashi M, Oba K and Takahashi Y: Clinical significance of serum tumor markers for gastric cancer: A systematic review of literature by the task force of the japanese gastric cancer association. Gastric Cancer 17: 26-33, 2014.
25. Sun DW, An L and Lv GY: Albumin-fibrinogen ratio and fibrinogen-prealbumin ratio as promising prognostic markers for cancers: An updated meta-analysis. World J Surg Oncol 18: 9, 2020.

26. Wu M, Pan Y, Jia Z, Wang Y, Yang N, Mu J, Zhou T, Guo Y, Jiang $\mathbf{J}$ and Cao $\mathrm{X}$ : Preoperative plasma fibrinogen and serum albumin score is an independent prognostic factor for resectable stage II-III Gastric Cancer. Dis Markers 2019: 9060845, 2019.

27. Li Y, Yang JN, Cheng SS and Wang Y: Prognostic significance of FA score based on plasma fibrinogen and serum albumin in patients with epithelial ovarian cancer. Cancer Manag Res 11: 7697-7705, 2019.

28. Zhang Y and Xiao G: Prognostic significance of the ratio of fibrinogen and albumin in human malignancies: A meta-analysis. Cancer Manag Res 11: 3381-3393, 2019.

29. Sun SY, Chen PP, Meng LX, Li L, Mo ZX, Sun CH, Wang Y and Liang FH: High preoperative plasma fibrinogen and serum albumin score is associated with poor survival in operable esophageal squamous cell carcinoma. Dis Esophagus: 32, 2019 doi: 10.1093/dote/doy057.

30. Zhang J, Li SQ, Liao ZH, Jiang YH, Chen QG, Huang B, Liu J, $\mathrm{Xu}$ YM, Lin J, Ying HQ and Wang XZ: Prognostic value of a novel FPR biomarker in patients with surgical stage II and III gastric cancer. Oncotarget 8: 75195-75205, 2017.

31. Batschauer AP, Figueiredo CP, Bueno EC, Ribeiro MA, Dusse LM, Fernandes AP, Gomes KB and Carvalho MG: D-dimer as a possible prognostic marker of operable hormone receptor-negative breast cancer. Ann Oncol 21: 1267-1272, 2010.

32. Tsimafeyeu IV, Demidov LV, Madzhuga AV, Somonova OV and Yelizarova AL: Hypercoagulability as a prognostic factor for survival in patients with metastatic renal cell carcinoma. J Exp Clin Cancer Res 28: 30, 2009.

33. Dolan RD, Laird BJA, Horgan PG and McMillan DC: The prognostic value of the systemic inflammatory response in randomised clinical trials in cancer: A systematic review. Crit Rev Oncol Hematol 132: 130-137, 2018.

34. Bilen MA, Martini DJ, Liu Y, Lewis C, Collins HH, Shabto JM, Akce M, Kissick HT, Carthon BC, Shaib WL, et al: The prognostic and predictive impact of inflammatory biomarkers in patients who have advanced-stage cancer treated with immunotherapy. Cancer 125: 127-134, 2019.

35. Schütte K, Tippelt B, Schulz C, Röhl FW, Feneberg A, Seidensticker R, Arend J and Malfertheiner P: Malnutrition is a prognostic factor in patients with hepatocellular carcinoma (HCC). Clin Nutr 34: 1122-1127, 2015.

36. Maasberg S, Knappe-Drzikova B, Vonderbeck D, Jann H, Weylandt KH, Grieser C, Pascher A, Schefold JC, Pavel M, Wiedenmann B, et al: Malnutrition predicts clinical outcome in patients with neuroendocrine neoplasia. Neuroendocrinology 104: $11-25,2017$

37. Palumbo JS, Talmage KE, Massari JV, La Jeunesse CM, Flick MJ, Kombrinck KW, Jirousková M and Degen JL: Platelets and fibrin(ogen) increase metastatic potential by impeding natural killer cell-mediated elimination of tumor cells. Blood 105 178-185, 2005.

38. Ridker PM, MacFadyen JG, Thuren T, Everett BM, Libby P and Glynn RJ: Effect of interleukin-1 $\beta$ inhibition with canakinumab on incident lung cancer in patients with atherosclerosis: Exploratory results from a randomised, double-blind, placebo-controlled trial. Lancet 390: 1833-1842, 2017.

39. Mantovani A: Cancer: Inflaming metastasis. Nature 457: 36-37, 2009

40. Greten FR and Grivennikov SI: Inflammation and cancer: Triggers, mechanisms, and consequences. Immunity 51: 27-41, 2019.

41. Kim S, Takahashi H, Lin WW, Descargues P, Grivennikov S, Kim Y, Luo JL and Karin M: Carcinoma-produced factors activate myeloid cells through TLR2 to stimulate metastasis. Nature 457: 102-106, 2009.

42. Marin Caro MM, Laviano A and Pichard C: Nutritional intervention and quality of life in adult oncology patients. Clin Nutr 26: 289-301, 2007

43. Savino $W$ and Dardenne $M$ : Nutritional imbalances and infections affect the thymus: Consequences on T-cell-mediated immune responses. Proc Nutr Soc 69: 636-643, 2010.

44. Spek CA, Versteeg HH and Borensztajn KS: Anticoagulant therapy of cancer patients: Will patient selection increase overall survival? Thromb Haemost 114: 530-536, 2015.

45. Wu CY, Wu MS, Kuo KN, Wang CB, Chen YJ and Lin JT: Effective reduction of gastric cancer risk with regular use of nonsteroidal anti-inflammatory drugs in Helicobacter pylori-infected patients. J Clin Oncol 28: 2952-2957, 2010. 
46. Klek S, Scislo L, Walewska E, Choruz R and Galas A Enriched enteral nutrition may improve short-term survival in stage IV gastric cancer patients: A randomized, controlled trial. Nutrition 36: 46-53, 2017.

47. Lin Y, Liu Z, Qiu Y, Zhang J, Wu H, Liang R, Chen G, Qin G, Li Y and Zou D: Clinical significance of plasma D-dimer and fibrinogen in digestive cancer: A systematic review and meta-analysis. Eur J Surg Oncol 44: 1494-1503, 2018.

48. Davalos D and Akassoglou K: Fibrinogen as a key regulator of inflammation in disease. Semin Immunopathol 34: 43-62, 2012.

49. Ferrigno D, Buccheri G and Ricca I: Prognostic significance of blood coagulation tests in lung cancer. Eur Respir J 17: 667-673, 2001.

50. Palumbo JS, Kombrinck KW, Drew AF, Grimes TS, Kiser JH, Degen JL and Bugge TH: Fibrinogen is an important determinant of the metastatic potential of circulating tumor cells. Blood 96: 3302-3309, 2000.

51. Im JH, Fu W, Wang H, Bhatia SK, Hammer DA, Kowalska MA and Muschel RJ: Coagulation facilitates tumor cell spreading in the pulmonary vasculature during early metastatic colony formation. Cancer Res 64: 8613-8619, 2004.

52. Yu X, Hu F, Yao Q, Li C, Zhang H and Xue Y: Serum fibrinogen levels are positively correlated with advanced tumor stage and poor survival in patients with gastric cancer undergoing gastrectomy: A large cohort retrospective study. BMC Cancer 16: 480, 2016.

53. Perisanidis C, Psyrri A, Cohen EE, Engelmann J, Heinze G, Perisanidis B, Stift A, Filipits M, Kornek G and Nkenke E: Prognostic role of pretreatment plasma fibrinogen in patients with solid tumors: A systematic review and meta-analysis. Cancer Treat Rev 41: 960-970, 2015.
54. Pfensig C, Dominik A, Borufka L, Hinz M, Stange J and Eggert M: A new application for albumin dialysis in extracorporeal organ support: Characterization of a putative interaction between human albumin and proinflammatory cytokines IL-6 and TNFa. Artif Organs 40: 397-402, 2016.

55. Diakos CI, Charles KA, McMillan DC and Clarke SJ: Cancer-related inflammation and treatment effectiveness. Lancet Oncol 15: e493-e503, 2014.

56. Paccagnella A, Morassutti I and Rosti G: Nutritional intervention for improving treatment tolerance in cancer patients. Curr Opin Oncol 23: 322-330, 2011.

57. Gupta D and Lis CG: Pretreatment serum albumin as a predictor of cancer survival: A systematic review of the epidemiological literature. Nutr J 9: 69, 2010.

58. Ouyang X, Dang Y, Zhang F and Huang Q: Low serum albumin correlates with poor survival in gastric cancer patients. Clin Lab 64: 239-245, 2018.

This work is licensed under a Creative Commons Attribution-NonCommercial-NoDerivatives 4.0 International (CC BY-NC-ND 4.0) License. 